\title{
Three-dimensional random walk models of individual animal movement and their application to trap counts modelling
}

\author{
D.A. Ahmed $^{\text {a,* }}$, S. Benhamou ${ }^{\text {b }}$, M.B. Bonsall ${ }^{\text {c }}$, S.V. Petrovskii ${ }^{\text {d,e }}$ \\ ${ }^{a}$ Center for Applied Mathematics and Bioinformatics (CAMB), Department of Mathematics and Natural Sciences, Gulf University for Science and Technology, P.O. Box 7207, \\ Hawally 32093, Kuwait \\ ${ }^{\mathrm{b}}$ Centre d'Ecologie Fonctionnelle et Evolutive, CNRS, Cogitamus Lab, Montpellier, France \\ ${ }^{\mathrm{c}}$ Mathematical Ecology Research Group, Department of Zoology, University of Oxford, Mansfield Road, OX1 3SZ OXford, UK \\ ${ }^{\mathrm{d}}$ School of Mathematics and Actuarial Science, University of Leicester, University Road, Leicester LE1 7RH, UK \\ ${ }^{\text {e }}$ Peoples Friendship University of Russia (RUDN University), 6 Miklukho-Maklaya St, Moscow 117198, Russian Federation
}

\section{A R T I C L E I N F O}

\section{Article history:}

Received 3 September 2020

Revised 11 April 2021

Accepted 15 April 2021

Available online 23 April 2021

\section{Keywords:}

3D random walks

Simple random walk

Correlated random walk

Biased random walk

Animal movement

Trapping

Optimal trap efficiency
Background: Random walks (RWs) have proved to be a powerful modelling tool in ecology, particularly in the study of animal movement. An application of RW concerns trapping which is the predominant sampling method to date in insect ecology and agricultural pest management. A lot of research effort has been directed towards modelling ground-dwelling insects by simulating their movement in 2D, and computing pitfall trap counts, but comparatively very little for flying insects with 3D elevated traps.

Methods: We introduce the mathematics behind 3D RWs and present key metrics such as the mean squared displacement (MSD) and path sinuosity, which are already well known in 2D. We develop the mathematical theory behind the 3D correlated random walk (CRW) which involves short-term directional persistence and the 3D Biased random walk (BRW) which introduces a long-term directional bias in the movement so that there is an overall preferred movement direction. In this study, we focus on the geometrical aspects of the 3D trap and thus consider three types of shape; a spheroidal trap, a cylindrical trap and a rectangular cuboidal trap. By simulating movement in 3D space, we investigated the effect of 3D trap shapes and sizes and of movement diffusion on trapping efficiency.

Results: We found that there is a non-linear dependence of trap counts on the trap surface area or volume, but the effect of volume appeared to be a simple consequence of changes in area. Nevertheless, there is a slight but clear hierarchy of trap shapes in terms of capture efficiency, with the spheroidal trap retaining more counts than a cylinder, followed by the cuboidal type for a given area. We also showed that there is no effect of short-term persistence when diffusion is kept constant, but trap counts significantly decrease with increasing diffusion.

Conclusion: Our results provide a better understanding of the interplay between the movement pattern, trap geometry and impacts on trapping efficiency, which leads to improved trap count interpretations, and more broadly, has implications for spatial ecology and population dynamics.

(C) 2021 The Author(s). Published by Elsevier Ltd. This is an open access article under the CC BY license (http://creativecommons.org/licenses/by/4.0/).

\section{Introduction}

Modelling individual animal movement and navigation strategies using random walks has long been a successful tradition in movement ecology (Nathan et al., 2008). The earliest models considered animal paths as uncorrelated and unbiased, e.g., Simple Random Walks (SRW) (Lin and Segel, 1974; Okubo, 1980). A natural extension known as the Correlated Random Walk (CRW), firstly conceived by Patlak (1953) and later developed by others (Hall, 1977; Kareiva and Shigesada, 1983; Bovet and Benhamou, 1988;
Benhamou, 2004), allows for correlation between the orientations of successive steps, resulting in a short term localized directional bias known as 'forward persistence'. This provides a more realistic description, as animals in the short term are more likely to keep moving in the same direction than to perform abrupt turns. Alternatively, a movement can show a consistent long term directional bias reflecting an overall preferred direction. This type of movement is known as a Biased Random Walk (BRW) (Marsh and Jones, 1988). If both short and long term biases are combined we obtain a Biased Correlated Random Walk (BCRW) (Benhamou, 2006; Codling et al., 2008; Bailey et al., 2018).

\footnotetext{
* Corresponding author.
} 
A tractable link between the 2D balanced CRW (i.e., left and right turns are equiprobable) and the mean squared displacement (MSD) was introduced by Tchen (1952) with constant step length, and later by Hall (1977) for variable step length. This helped bridge the gap between theory and field data, by providing a measure of the spatial spread of a population with the path length in terms of simple statistical moments. Kareiva and Shigesada (1983) further extended these results for a non-balanced 2D CRW. By comparing the observed MSD against that computed from theory, one could determine how well the CRW model predicted real animal movement (Weiss, 1994; Codling et al., 2008). This gave rise to a multitude of studies which successfully modelled the movement of a variety of species using the CRW, with many examples, including beetles (Byers, 2001), butterflies (Schultz and Crone, 2001), Elk (Morales et al., 2004; Fortin et al., 2005), grey seals (McClintock et al., 2012).

With cutting-edge developments in tagging and sensor technology, it is now possible to obtain accurate and refined 3D movement data, used to infer individual posture and heading (or 3D orientation). Measures of azimuthal, elevation and bank angles can be obtained through the usage of accelerometers and magnetometers, whereas, gyrometers can provide direct measures of rotations such as yaw, pitch and roll (Williams et al., 2020). Alongside this, there has been an increase in the number of studies which focus on 3D animal movements (Voesenek et al., 2016; Bras et al., 2017; de Margerie et al., 2018). In light of the above context, an extension to the results conceived by Hall (1977) to 3D is evidently due. Recently Sadjadi et al. (2015) and later Benhamou (2018) derived a mathematical expression for a key metric, namely, the MSD of the balanced CRW in 3D space, albeit using different mathematical techniques. This sets the stage for 3D CRWs and 3D BRWs to be tested as null models that could hypothetically provide a more realistic framework for swimming, burrowing and flying animals - due to the mere fact that movement is exercised in an additional (third) direction. Once the above movement models are formalised, these can then be used as a baseline for a theoretical insight into the dynamics of trap counts.

Trapping is the predominant sampling method in insect ecology and agricultural pest management. Their usage covers a wide scope of ecological scenarios, including but not limited to: general survey of insect diversity, detection of new invasive pests, delimitation of area of infestation, monitoring populations of established pests, or even as a direct control measure to suppress population build-up e.g., through mass trapping or 'lure and kill' eradication programs (Southwood, 1978; Radcliffe et al., 2008; El-Sayed et al., 2006; El-Sayed et al., 2009). Since their original conception, many traps have been designed for monitoring ground-dwelling insects with modifications to cater for particular species, habitats, environmental conditions and research requirements (MuirheadThomson, 1991), including recent efforts to standardize methodology by proposing a standard 2D pitfall trap design (Brown and Matthews, 2016). Alongside this, considerable progress has been made in modelling 2D pitfall trapping systems (Petrovskii et al., 2012; Ahmed and Petrovskii, 2019) even at multiple scales (Petrovskii et al., 2014). In contrast, very few attempts can be found in the literature which analyse 3D elevated or suspended traps (e.g., Byers, 2011; Byers, 2012), moreover, a well developed mathematical modelling framework is largely missing, and thus more research effort is required to help better understand the phenomena of trap count dynamics.

In agricultural field studies, a wide variety of trap designs are used for catching flying insects, and the trap type used often depends on the target species amongst other factors. For instance, fruit flies (Tephritidae) are known to be a serious invasive pest that cause considerable damage to a wide range of fruits and vegetables, leading to substantial economic losses (Qin et al., 2015;
Vargas et al., 2015). For this species, a common trap type is the red 'sticky' spherical trap where the trap surface is coated with an adhesive (Katsoyannos, 1989; Sivinski, 1990; Epsky et al., 2004; Daniel and Grunder, 2012). This specific design aims to mimic the size, shape and colour of a ripe apple, and is often baited with a synthetic fruit odour to lure the flies. Other types can be of different colours or shapes e.g., yellow/green cylindrical or rectangular cuboidal shape (Robacker and Rodriguez, 2004; Yee, 2019), see International Standard for Phytosanitary Measures (ISPM 26, 2018) for a detailed overview of 3D trapping scenarios, materials and procedures, also see (Epsky et al., 2004). From a mathematical perspective, these 'sticky' voluminous traps can be described as an enclosed shape with an absorbing surface. In addition to the aforementioned application in agriculture, it is also worth stating that in other contexts, the concept of trapping can be interpreted differently, such as in the case of encounters between arbitrarily moving organisms and immobile targets (synonymous to the trap) (Gurarie and Ovaskainen, 2013). The study of encounter rates is fundamental to many biological and ecological processes, e.g., cell-target encounter rates (Charnick and Lauffenburger, 1990) or predatorprey interactions of marine animals (Gerritsen and Strickler, 1977).

Firstly, we present the mathematical bases behind modelling individual animal movement paths in 3D, which can be utilised in various contexts. In particular, we describe mathematically a 3D SRW, and demonstrate how short/long term persistence mechanisms can be incorporated, for a more general and realistic 3D CRW or 3D BRW. Using the results from Benhamou (2018), we summarize important metrics, such as the MSD, and show how these RWs can be made equivalent in terms of diffusion. Secondly, using this 3D RW framework, we numerically simulate the movement of insects in 3D space, with a specific application focused on trapping. We reveal that trap counts vary non-linearly as a function of trap surface area or volume, and provide ansatz functions useful for trap count estimation. Furthermore, we investigate the interplay between the trap shape and elongation of 3D traps, the movement behaviour and how this can induce changes in trapping efficiency. More specifically, we analyse the impact of trap geometry and how short-term correlations ('micro-structure') or diffusion ('macro-structure') can affect capture rates. A better understanding of trap count dynamics and catch patterns lead to improved trap count interpretations, and more broadly, contribute towards applications in problems arising in contemporary spatial ecology.

\section{Methods}

\subsection{D random walks in Cartesian and spherical co-ordinates}

Individual animal movement can be modelled in 3D as a time series of locations $\mathbf{x}_{i}=\left(x_{i}, y_{i}, z_{i}\right)$ recorded at discrete times $t_{i}=\left\{t_{0}, t_{1}, t_{2}, \ldots\right\}$. The movement can therefore be seen as a series of discrete steps $\Delta \mathbf{x}_{i}=\mathbf{x}_{i}-\mathbf{x}_{i-1}$. Any 3D RW can be described in spherical coordinates, by expressing the step vector in terms of step lengths $l_{i}=\left\|\Delta \mathbf{x}_{i}\right\|$, azimuthal angle $\theta_{i}$ (equivalent to longitude) and polar angle $\phi_{i}$ (equivalent to co-latitude), using the transformation:

$\Delta x=l \cos (\theta) \sin (\phi), \quad \Delta y=l \sin (\theta) \sin (\phi), \quad \Delta z=l \cos (\phi)$,

$l \in[0, \infty), \quad \theta \in(-\pi, \pi], \quad \phi \in[0, \pi]$

The change of direction of an animal from heading $\left(\theta_{i}, \phi_{i}\right)$, between locations $\mathbf{x}_{i-1}$ and $\mathbf{x}_{i}$, to heading $\left(\theta_{i+1}, \phi_{i+1}\right)$, between locations $\mathbf{x}_{i}$ and $\mathbf{x}_{i+1}$, can be modelled as an orthodromic (or great circle) arc, characterized by two angles: the initial arc orientation $\beta_{i}$, measured between $-\pi$ and $\pi$ in the frontal plane with respect to the 
horizontal level, and the arc size $\omega_{i}$, measured between 0 and $\pi$ in the plane defined by the two headings:

$\omega_{i}=\cos ^{-1}\left[\cos \left(\phi_{i}\right) \cos \left(\phi_{i+1}\right)+\sin \left(\phi_{i}\right) \sin \left(\phi_{i+1}\right) \cos \left(\theta_{i+1}-\theta_{i}\right)\right]$

For a balanced CRW (including SRW as a special case) or BRW, the random variable $\beta$ is independent of $\omega$, and its distribution must also be centrally symmetric so that its mean sine and cosine are both null. Whether short or long term directional persistence is incorporated into the RW can be realised through the mean cosine of $\omega, c_{\omega}$ : one gets $c_{\omega}>0$ for a balanced CRW and BRW and $c_{\omega}=0$ for a SRW. CRW and BRW can be further distinguished based on how the heading at any step is determined. For both types of walks it is drawn at random around a predefined 3D direction $\boldsymbol{\mu}$. For a $\mathrm{CRW}, \boldsymbol{\mu}$ corresponds to the heading at the previous step, whereas for a BRW, $\boldsymbol{\mu}$ corresponds to the target direction. In this case, the arc size corresponding to the angular discrepancy between a given heading and the target direction will be referred to as $v$, which is statistically related to the arc size between successive headings $\omega$ through the relationship $c_{\omega}=c_{v}^{2}$ as occurs with 2D BRW (Marsh and Jones, 1988; Benhamou, 2006; Codling et al., 2008).

The Mean Squared Displacement (MSD), $\mathbb{E}\left[R_{n}^{2}\right]$, which is defined as the expected value of the squared beeline distance between an animals' initial and final locations after $n$ steps, serves as a useful metric to analyse movement patterns. The general MSD formulation for 2D CRW (Kareiva and Shigesada, 1983; Benhamou, 2006), in which left and right turns are not necessarily balanced, is extremely complex. We will consider here its extension in 3D space only for balanced CRW, developed by Benhamou (2018), and which reads:

$\mathbb{E}\left[R_{n}^{2}\right]=n \mathbb{E}\left[l^{2}\right]+2 \mathbb{E}\left[l^{2} \frac{c_{\omega}}{1-c_{\omega}}\left(n-\frac{1-c_{\omega}^{n}}{1-c_{\omega}}\right)\right.$,

also see Sadjadi et al. (2015) for an alternative derivation.

For a large step number $n$, the MSD approaches:

$\mathbb{E}\left[R_{n}^{2}\right]_{a}=n\left(\mathbb{E}\left[l^{2}\right]+2 \mathbb{E}[]^{2} \frac{c_{\omega}}{1-c_{\omega}}\right)=L \mathbb{E}[l]\left(\frac{1+c_{\omega}}{1-c_{\omega}}+\gamma^{2}\right)$,

where $L=n \mathbb{E}[l]$ is the mean path length and $\gamma=\sqrt{\frac{\mathbb{E}\left[l^{2}\right]}{\mathbb{E}\left[l^{2}\right.}-1}$ is the coefficient of variation of step length. For a 3D SRW, with $c_{\omega}=0$, the MSD reduces to $\mathbb{E}\left[R_{n}^{2}\right]=n \mathbb{E}\left[l^{2}\right]$, whatever the step number. It is readily seen from Eq. (4) that the MSD is asymptotically proportional to $n$, and therefore the walk becomes isotropically diffusive in the long term. The subscript ' $a$ ' is included here to represent the asymptotic value to which the MSD tends when $n$ increases indefinitely. For an isotropically diffusive RW, the MSD is related to the diffusion coefficient $D$ as follows: $\mathbb{E}\left[R_{n}^{2}\right]=2 q D T_{n}$ where $T_{n}$ is the duration of the $n$ step RW and $q=1,2,3$ corresponds to the number of dimensions (Crank, 1975; Turchin, 1998; Sornette, 2004; Codling et al., 2008). The amount of turning in a random search path can be quantified by the sinuosity index:

$S=\sqrt{\frac{S}{D}}=\sqrt{\frac{2 q n \mathbb{E}[l]}{\mathbb{E}\left[R_{n}^{2}\right]_{a}}}$

where $s$ is the mean speed, with $q=3$ for a random walk in 3D space (Benhamou, 2006; Benhamou, 2018).
In the case of a BRW, headings are drawn independently of each other in the target direction. This leads to the following expression for the MSD:

$$
\begin{aligned}
\mathbb{E}\left[R_{n}^{2}\right] & =n \mathbb{E}\left[l^{2}\right]+n(n-1) \mathbb{E}[l]^{2} c_{\omega} \\
& =\underbrace{n\left(\mathbb{E}\left[l^{2}\right]-\mathbb{E}\left[l^{2} c_{v}^{2}\right)\right.}_{\text {diffusion term }}+\underbrace{n^{2} \mathbb{E}[l]^{2} c_{v}^{2}}_{\text {advection term }} .
\end{aligned}
$$

This expression highlights that a BRW is essentially a combination of the diffusive random walk and a drift, and its MSD is dominated in the long-term by the contribution of the drift. It is worth noting that the MSD expressions for balanced CRW and BRW in 3D space are similar to those obtained in 2D space (Hall, 1977; Marsh and Jones, 1988). The only difference is that the mean cosine of turning angles that is used in 2D space is replaced by the mean cosine of orthodromic arcs corresponding to the reorientations between successive 3D headings.

We can derive the conditions under which two 3D balanced CRWs are 'equivalent', in the sense that they have the same MSD after $n$ steps, given that $n$ is sufficiently large. In particular, if we consider a SRW with step length $l^{*}$ and mean cosine $c_{\omega}^{*}=0$, assuming the same coefficient of variation of step length $\gamma$ and the same mean path length $L$, we obtain the following "condition of equivalence':

$\frac{\mathbb{E}\left[l^{*}\right]}{\mathbb{E}[l]}=\frac{1+\left(\frac{1-\gamma^{2}}{1+\gamma^{2}}\right) c_{\omega}}{1-c_{\omega}}$.

Now consider a SRW and a BRW with step lengths $l^{*}$ and $l^{\prime}$ and mean cosines $c_{\omega}^{*}=0$ and $c_{\omega}^{\prime}$, respectively. The condition of equivalence between these RWs in terms of diffusion is obtained with:

$\frac{\mathbb{E}\left[l^{*}\right]}{\mathbb{E}\left[l^{\prime}\right]}=1-\frac{c_{\omega}^{\prime}}{1+\gamma^{2}}$

\subsection{Mathematical bases for simulations of $3 D R W$}

We relied on a distribution of step length so that the distributions of increments $\Delta x, \Delta y$, and $\Delta z$, when reorientations are purely random (SRW), are zero-centred Gaussian distributions with the same standard deviation $\sigma$, which represents the mobility of the animal. For a SRW with such increments, the probability that the animal moves into an (infinitesimally) small vicinity of the current location $\mathbf{x}$, i.e within volume $d V=d \Delta x d \Delta y d \Delta z=l^{2} \sin (\phi) d l d \theta d \phi$, is:

$d P=\frac{l^{2}}{(\sigma \sqrt{2 \pi})^{3}} \exp \left(-\frac{l^{2}}{2 \sigma^{2}}\right) \sin (\phi) d l d \theta d \phi$.

As step length $l$, azimuth $\theta$ and polar angle $\phi$ are mutually independent random variables, one gets the following probability distribution functions for these variables:

$\lambda(l)=\frac{2 l^{2}}{\sigma^{3} \sqrt{2 \pi}} \exp \left(-\frac{l^{2}}{2 \sigma^{2}}\right), \quad \psi(\theta)=\frac{1}{2 \pi}, \quad \eta(\phi)=\frac{\sin (\phi)}{2}$.

The variable $\frac{l}{\sigma}$ follows a chi distribution with three degrees of freedom. Consequently, the mean step length is $\mathbb{E}[l]=\frac{4 \sigma}{\sqrt{2 \pi}}$ and mean squared step length $\mathbb{E}\left[l^{2}\right]=3 \sigma^{2}$. The coefficient of variation is therefore $\gamma=\sqrt{\frac{3 \pi}{8}-1}$. To specify the distributions of initial arc 
orientation $\beta$ and arc size $\omega$ in our simulations, we used the bivariate von Mises-Fisher distribution (vMF):

$v(\mathbf{z} ; \boldsymbol{\mu}, \kappa) d s=\frac{\kappa}{4 \pi \sinh (\kappa)} \exp (\kappa \boldsymbol{\mu} \cdot \mathbf{z}) d s$

where $\mathbf{z}=\left(z_{1}, z_{2}, z_{3}\right)$ is a unit random vector, $\boldsymbol{\mu}$ is a unit vector representing the mean direction, $\kappa>0$ is a measure of the concentration about the mean direction and $d s$ is the area of an infinitesimal surface element (Mardia et al., 1979). Given that $\boldsymbol{\mu}$ and $\mathbf{z}$ are two unit vectors which deviate by $\zeta$ from each other, one gets $\boldsymbol{\mu} \cdot \mathbf{z}=\cos (\zeta)$, with $\zeta=\omega$ for a balanced 3D CRW, where $\boldsymbol{\mu}$ corresponds to the previous heading, or $\zeta=v$ for a 3D BRW, where $\boldsymbol{\mu}$ corresponds to the target direction. Furthermore, by setting the pole of the sphere at the endpoint of $\boldsymbol{\mu}$, the area of the infinitesimal surface element $d s$ can be rewritten without loss of generality as $\sin (\zeta) d \beta d \zeta$ as it appears that $\zeta$ then behaves as a co-latitude and $\beta$ as a longitude. With $\beta$ uniformly distributed between $-\pi$ and $\pi$, one gets:

$\psi(\beta)=\frac{1}{2 \pi}, \quad \eta(\zeta ; \kappa)=\frac{\kappa}{2 \sinh (\kappa)} \exp (\kappa \cos (\zeta)) \sin (\zeta)$

where $\psi$ and $\eta$ correspond to the probability distribution functions of the initial arc orientation and arc size, respectively (Fisher et al., 1981; Mardia and Jupp, 2000). The mean cosine of $\zeta$ is given by:

$c_{\zeta}=\operatorname{coth}(\kappa)-\frac{1}{\kappa}, \quad \kappa>0$.

In the limit $\kappa \rightarrow 0$, the distribution of the arc size simplifies to $\eta(\zeta)=\frac{\sin (\zeta)}{2}$ with mean cosine $c_{\zeta}=0$, as expected for a SRW.

In the case of a SRW, the points are uniformly distributed on the whole surface. For increasing $\kappa$ values, the points are more concentrated towards the pole $\boldsymbol{\mu}$, see Fig. 1. Directional correlation is introduced to get a 3D balanced CRW, by randomly generating a heading from a distribution where the mean direction $\boldsymbol{\mu}$ corresponds to the previous heading, whereas a 3D BRW is obtained by randomly generating a heading from a distribution where the mean direction $\boldsymbol{\mu}$ corresponds to the target direction.

To tune the scale parameters of various CRW so that they are equivalent in terms of diffusion, we can express Eq. (7) as:

$\frac{\sigma^{*}}{\sigma}=\frac{1+\left(\frac{16}{3 \pi}-1\right) c_{\omega}}{1-c_{\omega}}$.

In the long term, a CRW with scale parameter $\sigma$ behaves as a SRW with scale parameter $\sigma^{*}$. The sinuosity of both walks can therefore be expressed as:

$S=2^{5 / 4} \pi^{-1 / 4}\left(\sigma^{*}\right)^{-1 / 2}$.

Similarly, for a BRW with step length distribution parameter $\sigma^{\prime}$ and long term persistence parameter $\kappa^{\prime}$ one gets: $\frac{\sigma^{*}}{\sigma^{\prime}}=1-\frac{8}{3 \pi} c_{v}^{2}$

with $c_{v}=\operatorname{coth}\left(\kappa^{\prime}\right)-\frac{1}{\kappa^{\prime}}$.

\subsection{Modelling trapping using numerical simulations}

In 3D trapping scenarios, consider a population of $N$ individuals moving independently of each other. The path of each individual is modelled as a 3D RW in unbounded space, with initial location $\mathbf{x}_{0}=\left(x_{0}, y_{0}, z_{0}\right)$ in proximity of a 3D trap. Each subsequent step is determined by the recurrence relation $\mathbf{x}_{i}=\mathbf{x}_{i-1}+\Delta \mathbf{x}_{i}$, resulting in a RW which is governed by the type of probability distribution for the step vector $\Delta \mathbf{x}$, and its properties. We assume that each walker moves until it is trapped or has travelled a path of length $L$, which can easily be converted into approximate (or average) time by considering the mean speed $s$. We introduce the concept of trapping by stating that at each step $i$, any individual which is within the confines of a trap is removed from the system, leading to trap counts or captures. Under such conditions, the trap surface is absorbing and the simulation allows cumulative trap counts $\mathfrak{I}$ to be recorded. In our simulations, we assume the absence of mortality or reproduction, so that the population at each step can only decrease, due to trapping, or otherwise remains stable. As an example, Fig. 2 shows the distribution of the individuals over the 3D space after performing the random walk of a given length $L$.

In this study, we consider three shapes of 3D traps, namely the spheroid, cylindrical and rectangular cuboid types with trap geometry $\mathfrak{D}$ defined by the following:

1. Spheroid (i.e., ellipsoid of revolution) trap with equatorial radius $r_{s}$ and polar radius $h_{s}$,

$$
\mathfrak{D}_{s}=\left\{(x, y, z) \mid \frac{x^{2}+y^{2}}{r_{s}^{2}}+\frac{z^{2}}{h_{s}^{2}}<1\right\},
$$

with the specific case $r_{s}=h_{s}$ reduces to a spherical shaped trap. 2. Cylindrical trap with radius $r_{c}$ and height $h_{c}$,

$$
\mathfrak{D}_{c}=\left\{(x, y, z)\left|x^{2}+y^{2}<r_{c}^{2},\right| z \mid<\frac{h_{c}}{2}\right\} .
$$

3. Rectangular cuboid trap with square base of side length $e_{b}$ and height $h_{b}$,

$$
\mathfrak{D}_{b}=\left\{(x, y, z)|| x\left|<\frac{e_{b}}{2},\right| y\left|<\frac{e_{b}}{2},\right| z \mid<\frac{h_{b}}{2}\right\},
$$

with the specific case $e_{b}=h_{b}$ reduces to a cube shaped trap. Subscripts ' $s, c, b$ ' refer to the spheroid, cylindrical, cuboid types, respectively. (a) $\kappa=0$

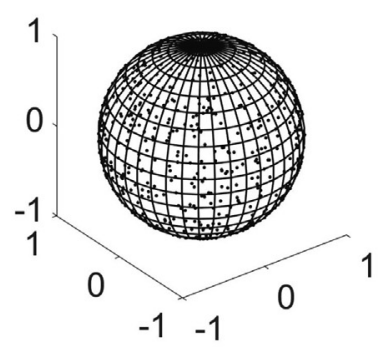

(b) $\kappa=5$

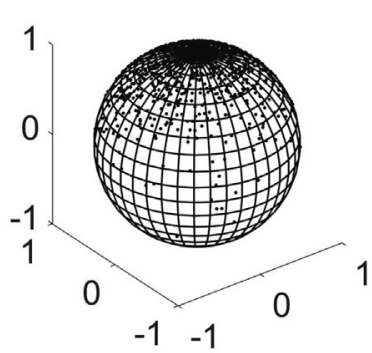

(c) $\kappa=20$

(d) $\kappa=100$

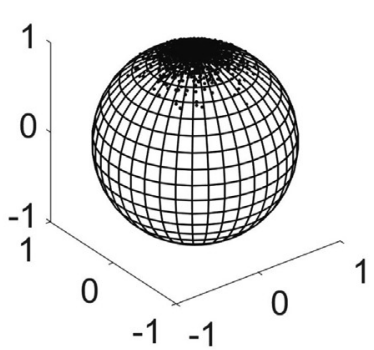

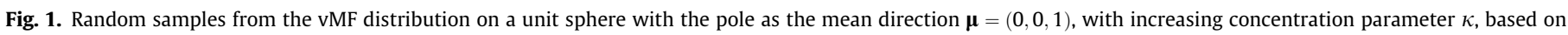
1000 simulated points. 
(a) $L=0$

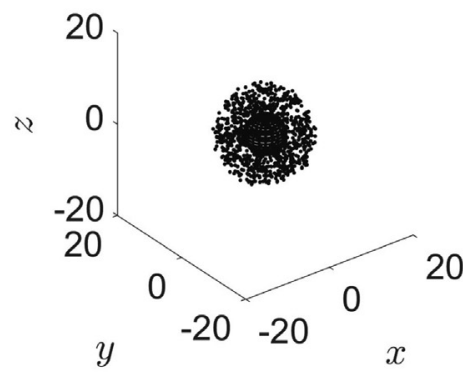

(d) $L=0$

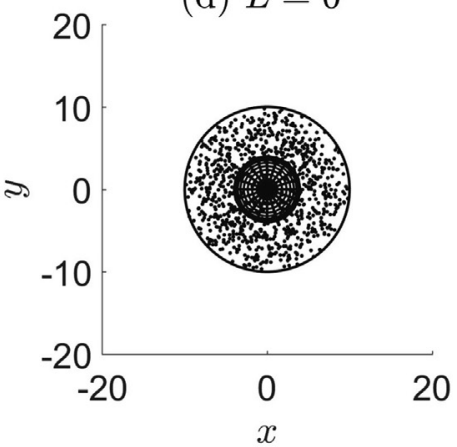

(b) $L=50$

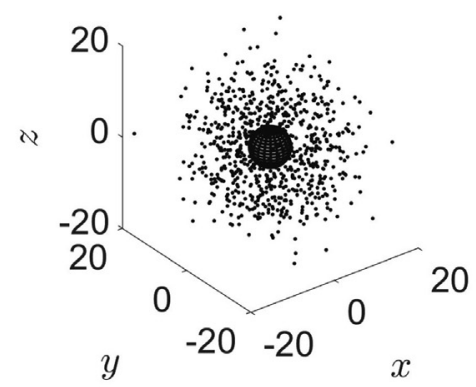

(e) $L=50$

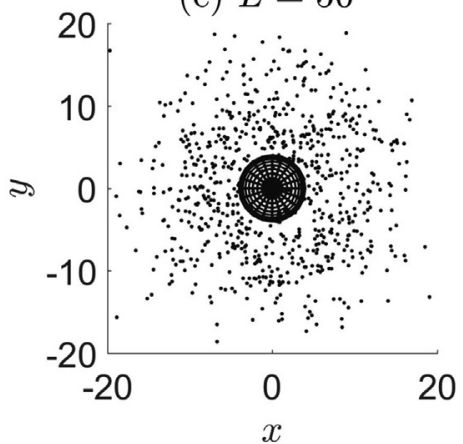

(c) $L=500$
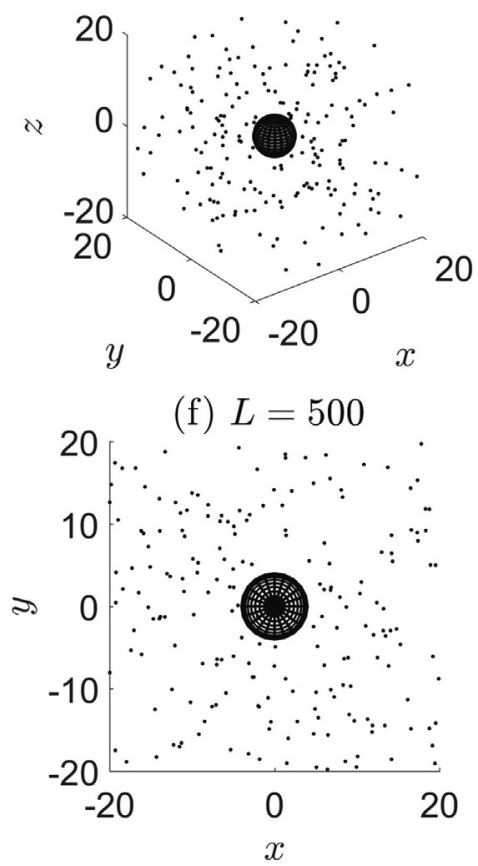

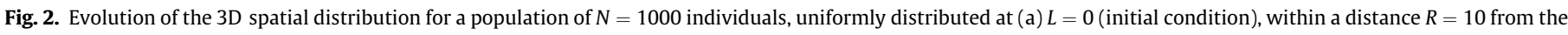

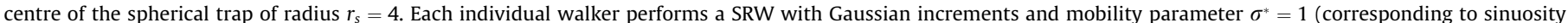

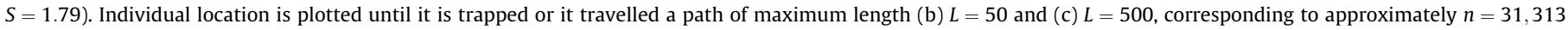

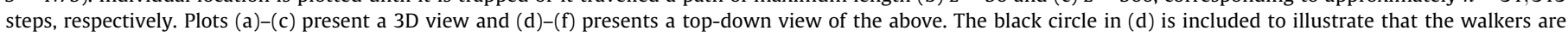
confined within the vicinity at $L=0$, but later move in unbounded space.

(a) Spheroid trap

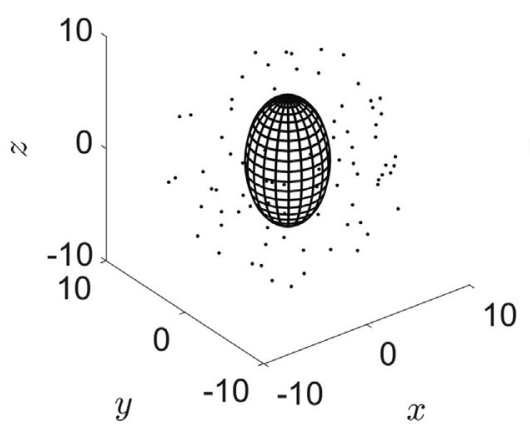

(b) Cylindrical trap

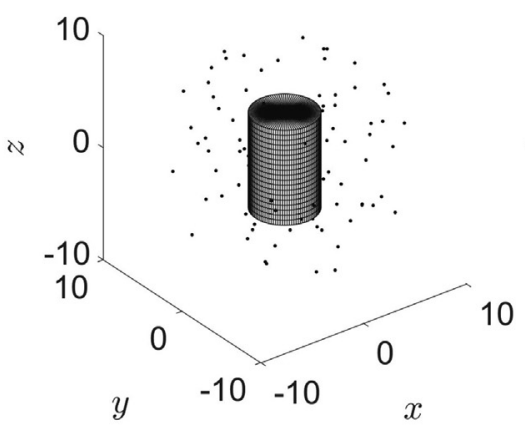

(c) Cuboid trap

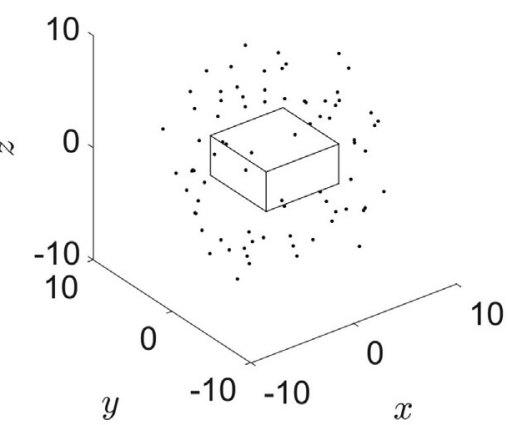

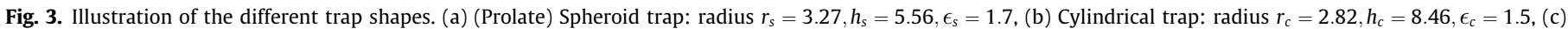

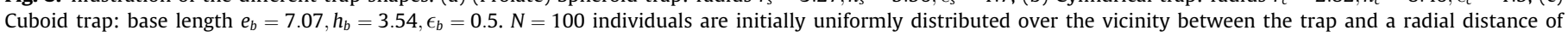

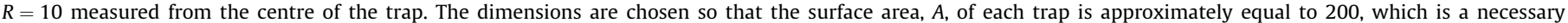
requirement to compare between these geometries (see explanation in §3.1).

For any trap type, we can specify its shape by introducing dimensionless elongation parameters. For the spheroid, we considered the ratio of polar to equatorial radii $\epsilon_{s}=\frac{h_{s}}{r_{s}}$, where $\epsilon_{s}<1$ corresponds to an oblate spheroid and $\epsilon_{s}>1$ to a prolate spheroid. For the cuboid we considered the ratio of height to base side length $\epsilon_{b}=\frac{h_{b}}{e_{b}}$, where $\epsilon_{b}=1$ corresponds to a cube, and for the cylinder we considered the ratio of height to base diameter $\epsilon_{c}=\frac{h_{c}}{2 r_{c}}$. (See Fig. 3)

We can then write expressions for the total surface area as:
$A_{s}=4 \pi r_{s}^{2} f\left(\epsilon_{s}\right)$ where $f\left(\epsilon_{s}\right)= \begin{cases}\frac{1}{2}\left[1+\frac{\epsilon_{s}^{2}}{\sqrt{1-\epsilon_{s}^{2}}} \tanh ^{-1}\left(\sqrt{1-\epsilon_{s}^{2}}\right)\right], & \epsilon_{s}<1 \\ 1, & \epsilon_{s}=1 \\ \frac{1}{2}\left[1+\frac{\epsilon_{s}^{2}}{\sqrt{\epsilon_{s}^{2}-1}} \sin ^{-1}\left(\frac{\sqrt{\epsilon_{s}^{2}-1}}{\epsilon_{s}}\right)\right], & \epsilon_{s}>1\end{cases}$

$A_{c}=2 \pi r_{c}^{2}\left(1+2 \epsilon_{c}\right), \quad A_{b}=2 e_{b}^{2}\left(1+2 \epsilon_{b}\right)$,

and for volume:

$V_{s}=\frac{4}{3} \pi \epsilon_{s} r_{s}^{3}, \quad V_{c}=2 \pi \epsilon_{c} r_{c}^{3}, \quad V_{b}=\epsilon_{b} e_{b}^{3}$. 
We can also express volume as a function of area as:

$V_{s}=\frac{\epsilon_{s}}{6 \sqrt{\pi}}\left(\frac{A_{s}}{f\left(\epsilon_{s}\right)}\right)^{\frac{3}{2}}, \quad V_{c}=\frac{\epsilon_{c}}{\sqrt{2 \pi}}\left(\frac{A_{c}}{1+2 \epsilon_{c}}\right)^{\frac{3}{2}}, \quad V_{b}=\epsilon_{b}\left(\frac{A_{b}}{2\left(1+2 \epsilon_{b}\right)}\right)^{\frac{3}{2}}$.

The initial distribution of individual location is considered to be uniform over a vicinity, which is defined as the space between the trap and some fixed outer distance $R$, measured from the centre of the trap. This accounts for individuals having reached the vicinity of the trap from a further distance but not yet trapped, as intrinsically random. In the case of a spherical trap, we can think of this as the 3D extension of uniformly distributed points on an annulus in $2 \mathrm{D}$, i.e., between two concentric spheres (or as a spherical shell). If we describe initial location in spherical co-ordinates as $\mathbf{x}_{0}=\left(r_{0}, \theta_{0}, \phi_{0}\right)$, then the corresponding probability density functions can be written explicitly as:

$\mathfrak{R}\left(r_{0}\right)=\frac{3 r_{0}^{2}}{R^{3}-r_{s}^{3}}, \quad \Theta\left(\theta_{0}\right)=\frac{1}{2 \pi}, \quad \Phi\left(\phi_{0}\right)=\frac{\sin \phi_{0}}{2}$.

Using the inverse transform technique (Grimmet and Stirzaker, 2001), the initial location of each individual can then easily be simulated by:

$\mathbf{x}_{0} \sim\left(\sqrt[3]{\left(R^{3}-r_{s}^{3}\right) U_{1}+r_{s}^{3}}, 2 \pi U_{2}, \cos ^{-1}\left(1-2 U_{3}\right)\right)$

where $U_{1}, U_{2}$ and $U_{3}$ are independent random variables drawn from the uniform distribution between 0 and 1 .

In the case of other trap shapes, the vicinity no longer has an infinite number of symmetry axes and therefore, to simulate a homogeneous population is not as straightforward. In these cases, we drawn the initial locations at random in the whole sphere of radius $R$, and removed those occurring within the trap.

\section{Results}

\subsection{Effect of trap shape type}

Consider the usual simulation setting outlined in $\S 2.3$, for a spherical trap $\left(\epsilon_{s}=1\right)$ with increasing trap size, see Fig. 4. By simulating trap count data for different sized spherical traps, we can investigate whether captures are better correlated with trap surface area or trap volume. This approach can also be applied to cuboid and cylindrical traps. We considered a cube trap $h_{b}=e_{b}$ $\left(\epsilon_{b}=1\right)$, and a 'normalized' cylinder where the height is equal to the base diameter $h_{c}=2 r_{c}\left(\epsilon_{c}=1\right)$. The normalized cylinder and the cube lie within a sphere of radius $R$ provided that the following inequalities apply:

$r_{c}<\frac{R}{\sqrt{2}}, \quad A_{c}<3 \pi R^{2}, \quad V_{c}<\frac{\pi R^{3}}{\sqrt{2}}$

$e_{b}<\frac{2 \mathrm{R}}{\sqrt{3}}, \quad A_{b}<8 R^{2}, \quad V_{b}<\frac{8 R^{3}}{3 \sqrt{3}}$,

which we use to determine the range of trap dimensions, areas and volumes.

The simulated trap counts are shown in Fig. 5. It is readily seen that the cumulative trap count is a monotonously increasing, nonlinear function of trap surface area and volume. Note that the order of trap shapes, in terms of capture efficiency, is reversed depending on whether we consider the traps to have equal total area or volume.

\subsection{Effect of trap elongation}

In the following, we investigate the variation in trap counts for different configurations of spheroidal, cylindrical and cuboid traps, assuming the same total surface area or volume. Trap counts for a

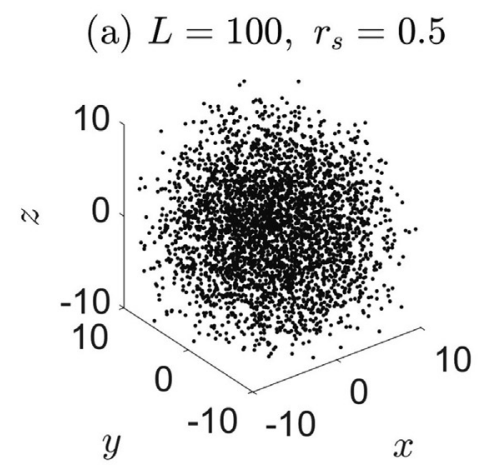

$$
\text { (d) } L=500, r_{s}=0.5
$$

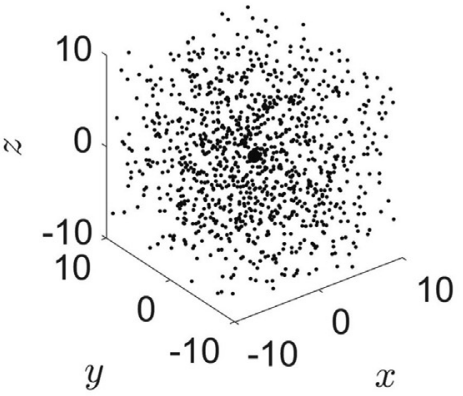

(b) $L=100, r_{s}=2$

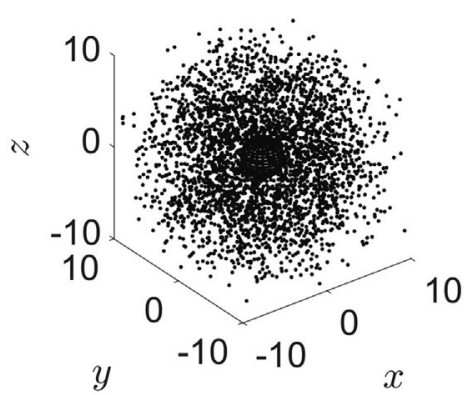

(e) $L=500, r_{s}=2$

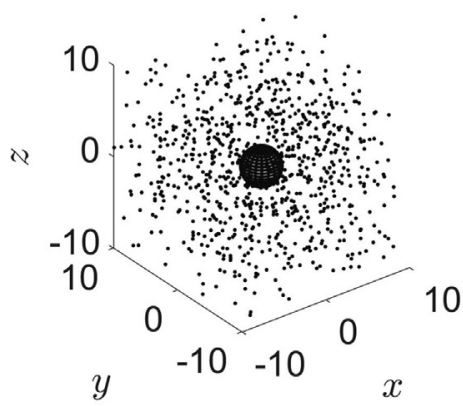

(c) $L=100, r_{s}=4$

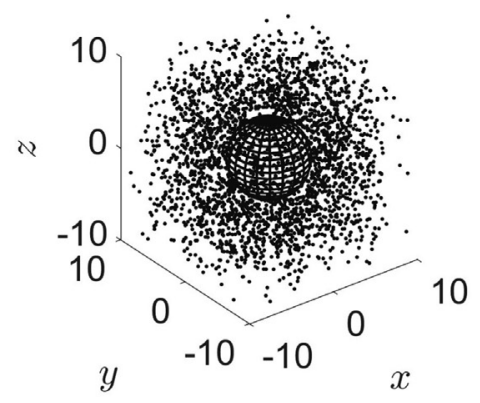

(f) $L=500, r_{s}=4$

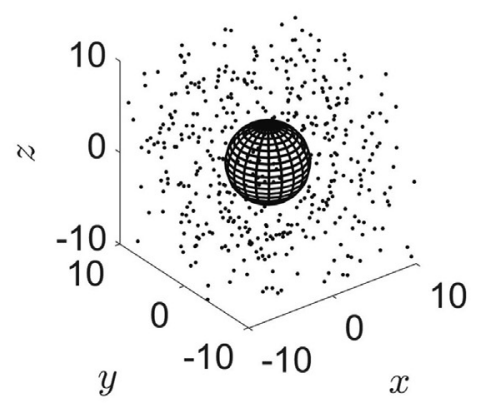

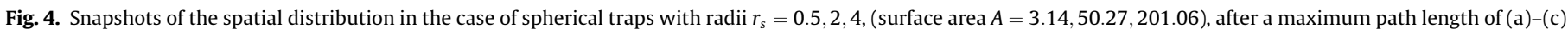
$L=100$ and (d)-(f) $L=500$ has been reached. Each individual executes a SRW in unbounded space with mobility parameter $\sigma^{*}=1(S=1.79)$. 

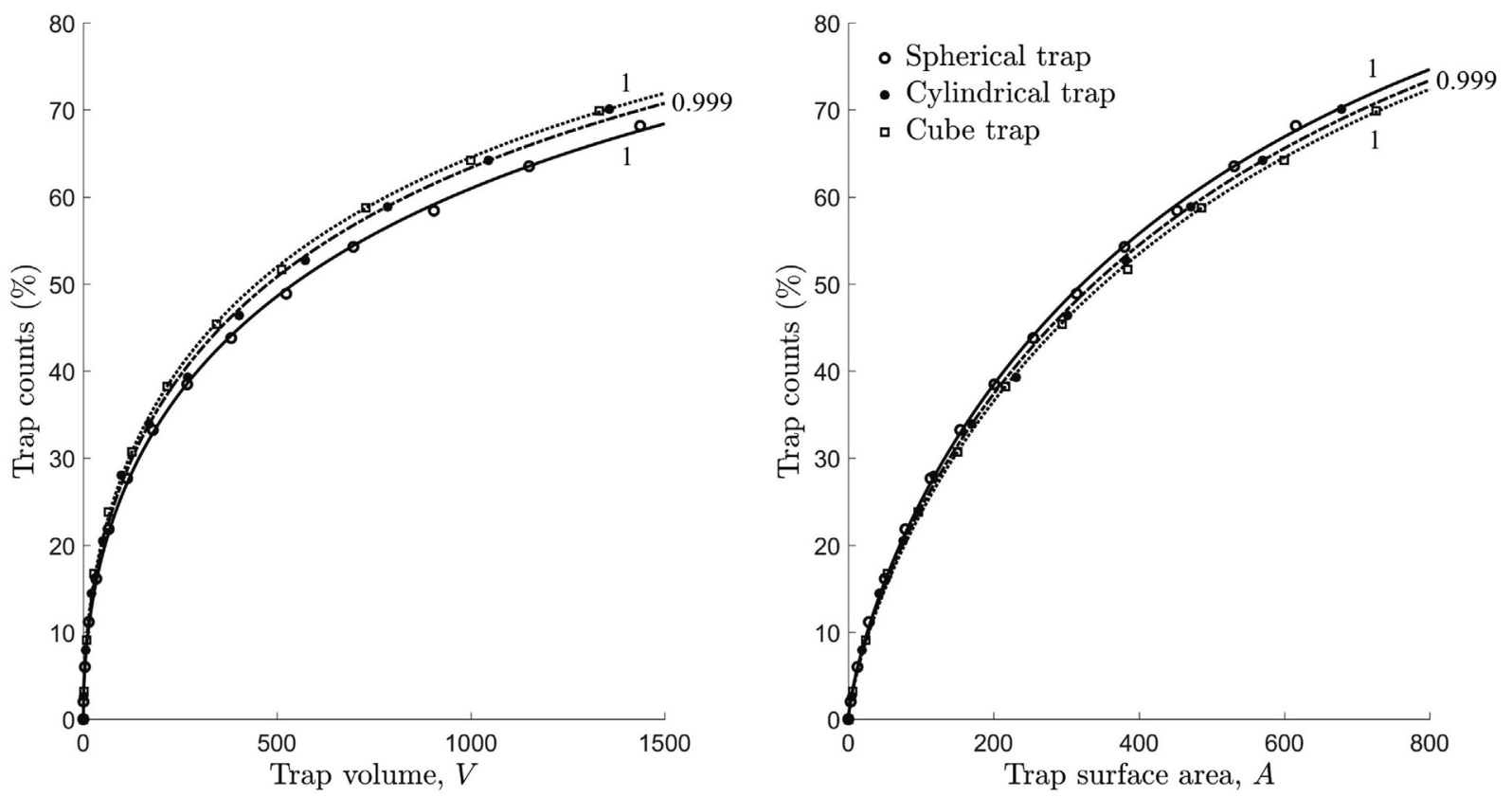

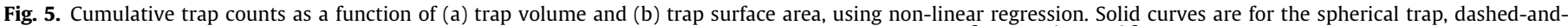

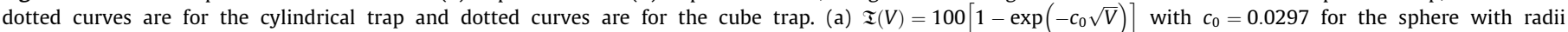

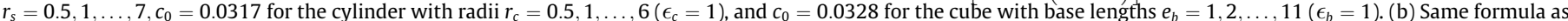

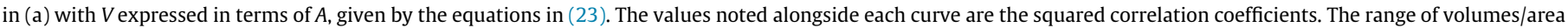

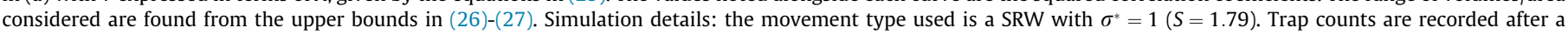
maximum path length of $L=500$ has been reached.
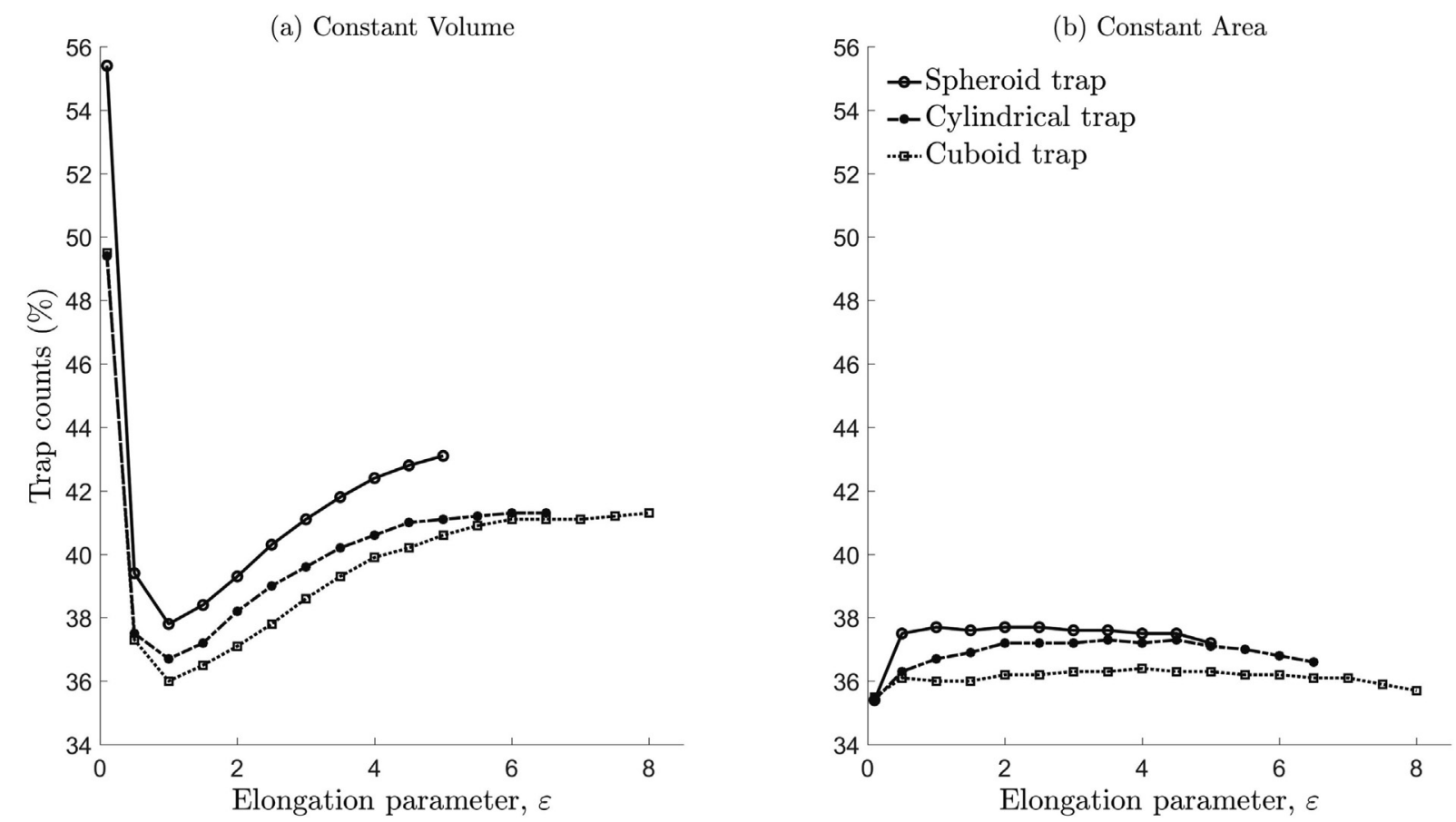

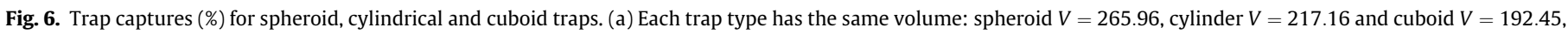

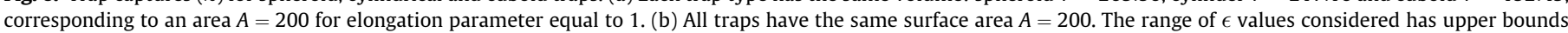

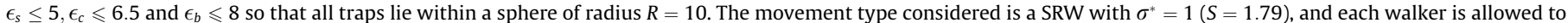
travel up to a maximum path length $L=500$.

given volume and a given trap shape (Fig. 6a) varies a lot, but the variation as a function of the elongation parameter is mainly due to a variation of area. Indeed, the sharp increase in the trap count seen in Fig. 6a for small $\epsilon$ is an immediate consequence of the fact that the decrease in $\epsilon$ to values $\epsilon \ll 1$ makes the shape almost flat. In order to preserve the volume, the area then becomes large. On the contrary, when the area is kept constant for all trap shape types and elongation parameter, we found that the number of captures 

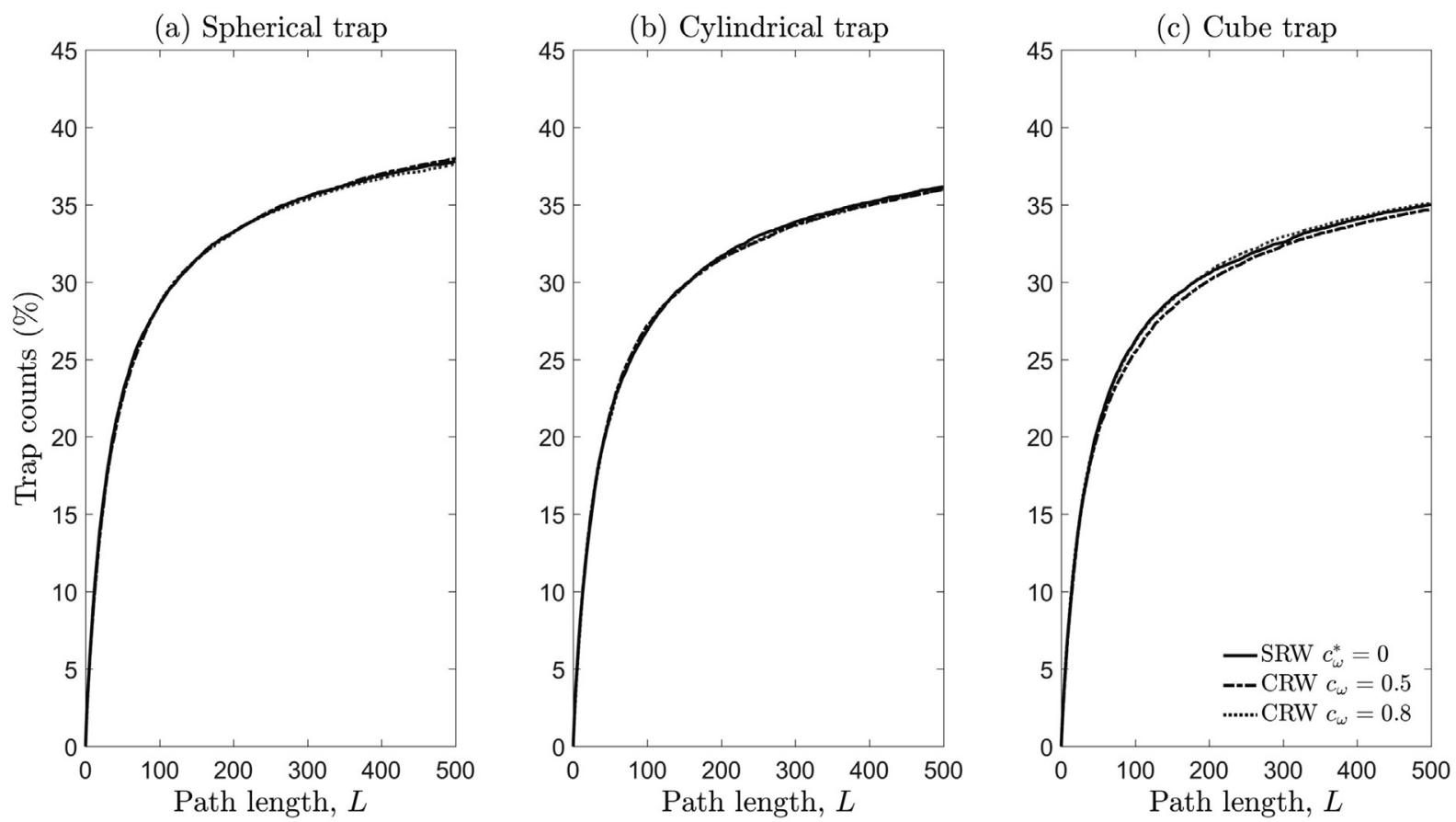

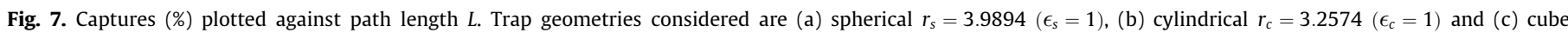

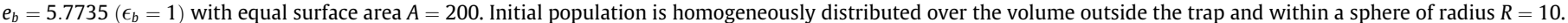

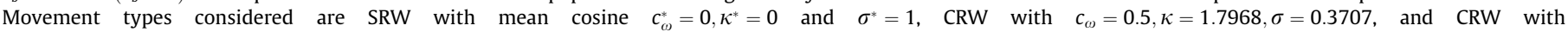

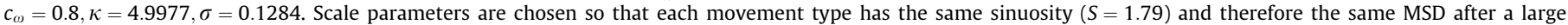
number of steps for a given path length (see Eqs. (14) and (15)).

does not vary much (Fig. 6b). In this context, spheroidal traps slightly outperform cylindrical and cuboid traps in terms of capture efficiency. As elongation has no noticeable effect (for each type of trap) whereas this factor changes the volume for a given area, it makes sense to consider traps with the same area for subsequent analyses of the possible effects of short-term persistence, long-term directional bias and diffusion of the walk.

\subsection{No effect of short-term persistence when diffusion is kept constant}

Fig. 7 demonstrates that the inclusion of short-term persistence results in identical trap counts, assuming that all individuals perform a path with the same diffusion and same maximum path length irrespective of trap geometry.

\subsection{Effect of diffusion}

Fig. 8 confirms that spherical traps are, on average, the most efficient. Trap counts decrease with increasing diffusion, as soon as the maximum path length is sufficiently long. We observe small but noticeable differences in efficiencies on comparing the cube and cylindrical traps. This indicates that the impact of trap geometry can be important in this case. Also, we note that trap counts accumulate much slower if diffusion is low, and given that the path length is small. This has an intuitive interpretation that individuals, on average, do not have enough time to approach the trap.

Fig. 9 shows that trap counts decrease, on average, with increasing mean cosine (i.e., increasing short-term persistence/diffusion), for all trap shapes. It is worth noting that, when diffusion is large, trap count differences decrease, implying that the impact of trap geometry is then not that important. For relatively smaller values of diffusion, there is a clear hierarchy of trap shape in terms of trapping efficiency, with the spherical trap retaining the most counts, followed by the cylindrical trap, and then the cube.

\subsection{Effect of long-term bias}

Fig. 10 shows that the presence of long-term bias towards the trap, as expected, dramatically increases captures. There is a clear hierarchy of trap shapes in terms of capture efficiency.

\section{Discussion}

Dispersal and movement are fundamental for understanding the distribution and abundance of species in ecosystems. All species change their location in space at least during some stages of their life. Movement is known to have fundamental implications for individual survival, behaviours and reproduction, the population dynamics, and on fitness and evolution (Clobert et al., 2001; Bullock et al., 2002). The capacity for movement is prolific across different species. For instance, while plants do not normally move, their seeds and spores do and can cover considerable distances before settling down. Insect eggs and pupae do not move, but larvae and/or adults move most of the time, e.g., to forage for food. Most vertebrates move practically all their life, e.g., to forage, to avoid predators, to look for a mating partner, etc. Understanding of the typical movement patterns is therefore a major focus of ecology and population biology (Okubo, 1980; Pyke, 1984; Turchin, 1998; Nathan et al., 2008; Owen-Smith et al., 2010; Viswanathan et al., 2011; Pyke, 2015).

Among many research tools available to study individual animal movement, mathematical modelling plays an increasingly important role (Turchin, 1998; Codling et al., 2008; Johnson et al., 2008; Wilson et al., 2013; Edelhoff et al., 2016). Random walks (RWs) are appropriate approaches for understanding species movement patterns particularly as a stochastic or statistical description of dispersal. They are easy to implement: it is rather straightforward to investigate movement paths using computer simulations based on RWs. More importantly, by considering individual movement as a stochastic process, it is often possible to 

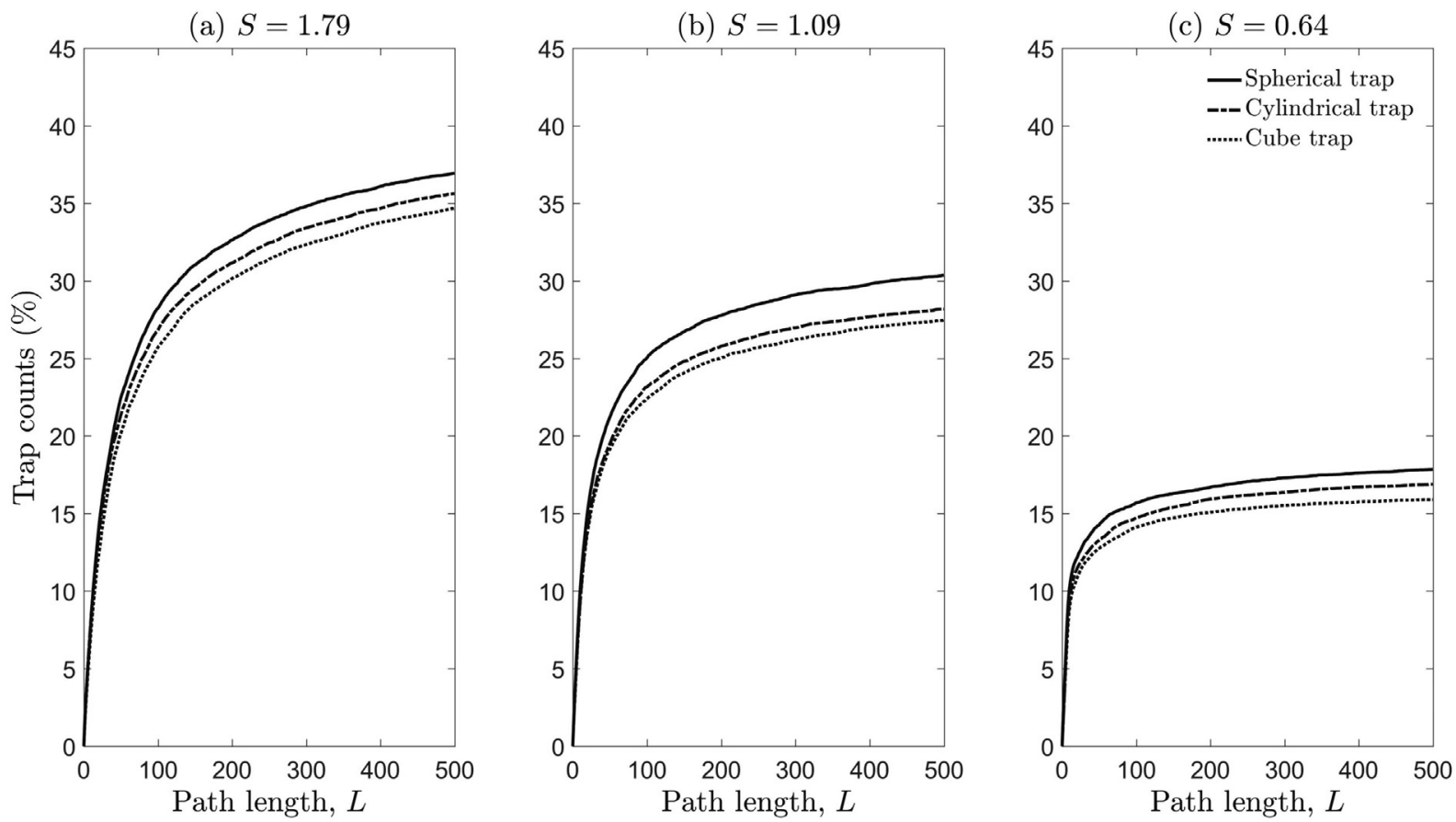

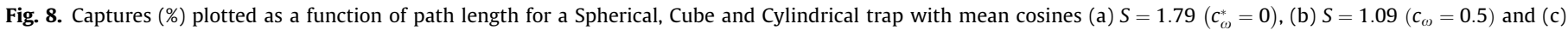
$S=0.64\left(c_{\omega}=0.8\right)$. Contrary to what occurs in Fig. 7, the scaling parameter was the same for all walks $\left(\sigma^{*}=\sigma=1\right)$ so that the diffusion increases with $c_{\omega}$.

obtain a general analytical description, in terms of the dispersal kernel and/or the statistical moments, as functions of time, and thus to reveal generic properties of different movement behaviours (Reynolds, 2010; Codling and Plank, 2011; James et al., 2011; McClintock et al., 2012; Tilles and Petrovskii, 2015; Tilles et al., 2017). While there has recently been considerable progress in understanding these issues, most theoretical studies on animal movement have been predominantly limited to 2D cases. Meanwhile, in the real-world application of monitoring flying insects (e.g., different taxa of fruit flies), traps are usually elevated above the ground, sometimes at a significant height, for e.g., 1-10 metres
(Epsky et al., 2004). Thus, the movement of flying insects in the vicinity of an elevated trap is essentially performed in 3D space, and hence it should be modelled as such.

Understanding the efficiency of trapping resulting from the interplay between the movement pattern (as described by the SRW, CRW and BRW) and the shape of the trap was the focus of this study. We first derived the expression for the MSD in terms of the number of steps (or as a function of time), and conditions of equivalence between RWs with different step size distributions were obtained in terms of diffusion. We then proceeded to numerical simulations of trap counts with traps of different shapes com-

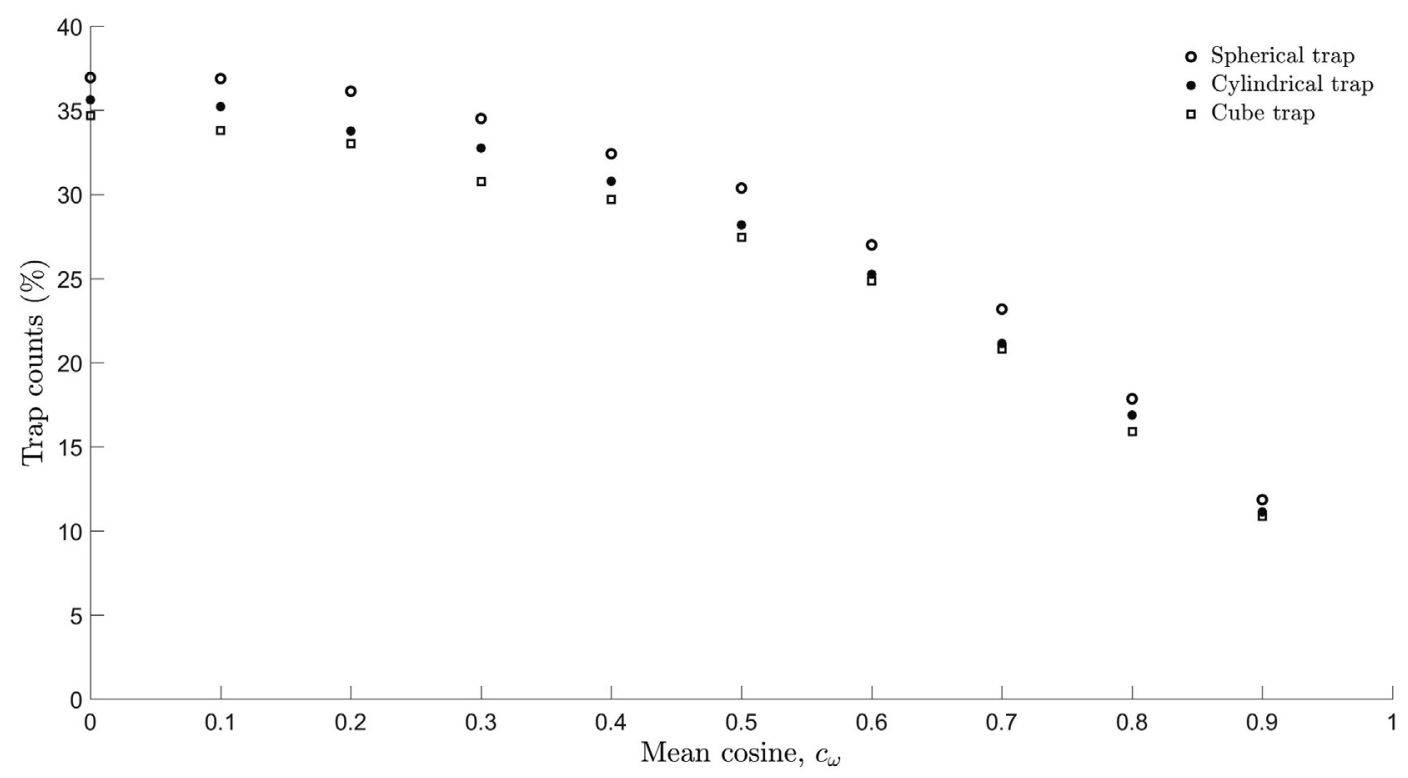

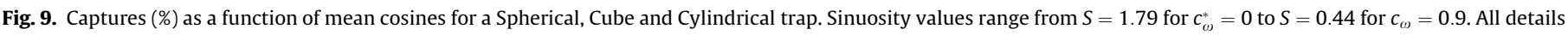
are the same as that described in the caption of Fig. 8. 


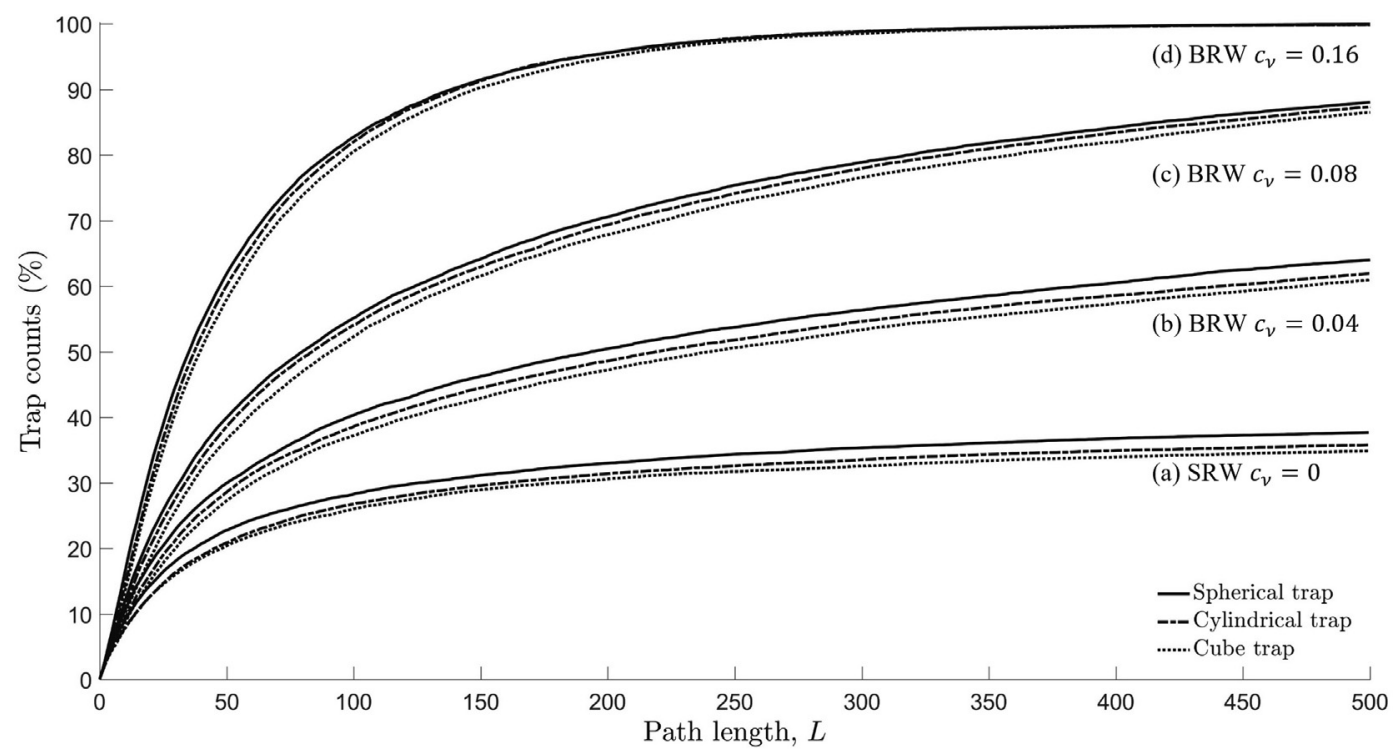

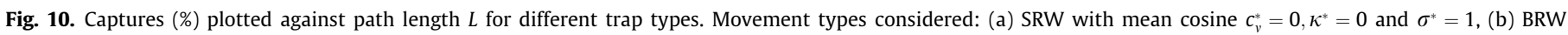

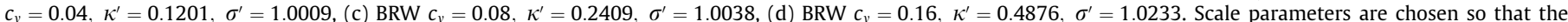
BRW is asymptotically equivalent to a SRW in terms of diffusion. All other details, such as trap dimensions, are exactly the same as that used for Fig. 8.

monly used in ecological studies, i.e., spheroid, cylinder and cuboid. As one result of immediate practical importance, we revealed the non-linear dependence of trap counts on the geometry of traps, quantified by either the area of the trap surface or the trap volume, and provided corresponding ansatz functions useful for trap count estimations (see Fig. 5). On considering trap elongation, we found that trap counts do not vary much given that the surface area is fixed, and that there is a clear hierarchy in terms of which traps are more efficient, with the spheroidal trap outperforming the cylindrical trap, followed by the cuboidal trap (see Fig. 6). Also, rather counter-intuitively, we showed that the short-term persistence of the individual movement ('micro-struc ture') does not have any notable effect on the trap counts when the diffusion is kept constant (see Fig. 7), and it turns out that only the 'macro-structure' is important (see Figs. 8 and 9). Although we found small but noticeable differences in trap counts on considering a single trap, in real field experiments several traps (or clusters of traps) are used in some specific configurations, such that the density of traps and their positioning plays a role in the catches, and therefore under such a scenario, the trap geometry can have a significant impact on the number of captures.

One application of movement models arises from the needs of ecological monitoring (Greenslade, 1964; Byers, 2012; Siewers et al., 2014; Miller et al., 2015). Monitoring of invertebrates, insects in particular, is often performed by installing traps and then interpreting trap counts (catches). The latter, however, appears to be a challenging problem. It is deceptively easy to interpret the trap counts in the relative way, i.e., 'larger count implies larger population', but this can be misleading or simply wrong because of the interplay between the movement activity and the population density: a small population of fast moving animals can result in the same trap count as a large population of slower moving animals (cf. 'activity-density paradigm' (Thomas et al., 1998)). An absolute interpretation of trap counts relating them to the population density in the vicinity of the trap is possible (Petrovskii et al., 2012; Petrovskii et al., 2014; Ahmed and Petrovskii, 2019) but it requires a succession of several trap counts and some information about the movement pattern such as the frequency distribution of step sizes and turning angles along the path (also the distribution of different movement modes, rest time, etc., in case of more complicated movement behaviours) as well as a good understanding of the effect of trap geometry (Ahmed and Petrovskii, 2019).

Furthermore, in the statistical application of models to ecological data, a pervasive and recurrent problem is understanding the biases introduced through the measurement or observation of the ecological system (e.g., Hilborn and Mangel, 1998; de Valpine and Hastings, 2002). More accurate estimates of the number of individuals that move or are present in a given location require the use of mathematical tools. Many distance sampling methods have been developed (Buckland et al., 2015) to link observations on counts of individuals to estimates of population size. More recently Bayesian hierarchical methods (e.g., Doucet et al., 2001; Bonsall et al., 2014; Kantas et al., 2015; Bonsall et al., 2020) have been developed and applied in an ecological context to approach the decomposition of error into measurement and process components. There are also several recent examples of frequentist methods being used for state-space, partially observed Markov process, and hidden Markov models in ecology to approach the decomposition of error (Fukaya and Royle, 2013; Li and Bolker, 2017; McClintock et al., 2020). The mathematical framework we develop here, provide a richer set of tools to be able to relate how the biases in individual behaviours influence measurement error problems and hence provide more robust determinants of population level measures. With a more detailed understanding of the effects of different trap geometries on capturing/detecting individuals in a population will provide more robust ways in which to discern broad scale ecological patterns.

Coupled movement and dynamical models such as integrodifference approaches (Kot and Schaffer, 1986; Lutscher, 2019) have widespread application in ecology for understanding invasion speeds (e.g., Kot, 1992), Allee effects (e.g., Wang et al., 2002), climate change (e.g., Zhou and Kot, 2011) and invasive species control (e.g., Kura et al., 2019). All rely on a dispersal kernel to relate movement from one location to another (e.g., Reimer et al., 2016; Reimer et al., 2017) and the influence this has on the population dynamics. This dispersal kernel is critical for ensuring model predictions can be accurately validated against experiments and/or observations. Our work on 3D RWs now provides a way in which to scale up from 
individual movement rules to generate appropriately formulated dispersal kernels. Furthermore, the individual basis to the movement and dispersal patterns provides an alternative approach to link movement and the population dynamics without recourse to simpler mean-field approaches.

A question may arise as to why one should use RWs to model explicitly hundreds or thousands of randomly moving animals rather than the corresponding mean-field mathematical description instead. If the potential ecological applications of our work is somewhat obvious, several methodological questions remain unresolved. It is well-known that, for the SRW, the dynamics of the population density distribution over space is described by the diffusion equation (Okubo, 1980; Skellam, 1951; Kareiva and Shigesada, 1983) and for the CRW, by the Telegraph equation, (e.g., see Turchin, 1998; Codling et al., 2008). However, note that the analytical solution of the diffusion equation, from which the average trap count can be calculated (see Petrovskii et al., 2012; Petrovskii et al., 2014; Ahmed and Petrovskii, 2015; Ahmed et al., 2018 ) is, even in case of relatively simple trap shapes such as a spheroid or cylinder, only available as a Fourier series where the exponents (the eigenvalues of the corresponding boundary problem) still need to be found numerically. With the reliancy on numerical approximations and approaches, the 'analytical' description of trap counts is not much different from that derived from the individual based model. In the case of more realistic movement described by the CRW, the situation is actually much more complex, as the solutions of the boundary problem for the Telegraph equation in the general case are not positively defined (Tilles and Petrovskii, 2019). Simulation of trap counts using individual based models therefore provides a robust and plausible alternative to analytical approaches.

Finally, we mention that there do exist modelling frameworks alternative to the discrete-time RW used in our study. Movement can be described in continuous time in terms of stochastic differential equations (SDEs) (Gurarie and Ovaskainen, 2011); this may potentially provide a more analytically tractable approach to study trap counts. A BRW can be written in terms of the OU process, whose associated Fokker-Planck equation (FPE) is a linear partial differential equation. Encounter rates can then be worked out from the probability density function of the individual position (e.g., see Martinez-Garcia et al. (2020)) and trap counts can be obtained by solving the FPE with absorbing boundary conditions at the trap surface (cf. Redner (2001)). Exploring the potential of these approaches as alternative tools to investigate the effect of movement properties and the shape and size of the trap on trap counts should become a focus of future research. We, however, mention here that solving SDEs or FPE in a non-trivial 3D geometry (as determined by the trap shape) is likely to require numerical simulations that can be algorithmically more complicated than the rather straightforward individual-based RW simulations.

\section{Conclusion}

In conclusion, these issues notwithstanding, we have shown how different trap geometries and the 3D movement of individuals can bias trapping efficiency. Understanding how diffusion, directed movement and trap shape can affect counts, estimates and observations has critical implications for spatial ecology and for understanding the distribution and abundance of species. These individual based, geometric approaches warrant further investigation and application in problems arising in contemporary spatial ecology. The next natural step that we hope to see in the near future, is analyses of real flying animal movements using 3D RW models.

\subsection{Abbreviations}

3D: Three-dimensional; SRW: Simple random walk; CRW: Correlated random walk; BRW: Biased random walk.

\subsection{Author contributions}

DAA and SB participated in the design of the study, conducted the simulations, analysed the results, drafted and critically revised the manuscript. MBB and SVP participated in the interpretation and discussion of results and also critically revised the manuscript. All authors gave final approval for publication and agree to be held accountable for the work performed therein.

\subsection{Funding}

This work was funded by the Kuwait Foundation for the Advancement of Sciences (KFAS) [Grant No.: PR1914SM-01, to DAA], the Gulf University for Science and Technology (GUST) internal seed fund [Grant No.: 187092, to DAA] and by the UK Engineering and Physical Sciences Research Council (EPSRC) [Grant No.: EP/ T027371/1, to SP]. This paper has been supported by the RUDN University Strategic Academic Leadership Program (to SP).

\subsection{Ethical Approval and Consent to participate}

Not applicable.

\subsection{Consent for publication}

Not applicable.

\subsection{Availability of supporting data}

Not applicable.

\section{Declaration of Competing Interest}

The authors declare that they have no known competing financial interests or personal relationships that could have appeared to influence the work reported in this paper.

\section{Acknowledgements}

DAA is thankful to Philip Maini (Oxford, UK) for a fruitful and stimulating discussion which helped improve the manuscript.

\section{References}

Ahmed, D., Petrovskii, S., 2015. Time dependent diffusion as a mean field counterpart of Lévy type random walk. Math. Model. Nat. Phenom. 10 (2), 5-26.

Ahmed, D., Petrovskii, S., 2019. Analysing the impact of trap shape and movement behaviour of ground-dwelling arthropods on trap efficiency. Methods Ecol. Evol. 10 (8), 1246-1264.

Ahmed, D., Petrovskii, S., Tilles, P., 2018. The Lévy or diffusion controversy: how important is the movement pattern in the context of trapping? Mathematics 6 (77).

Bailey, J., Wallis, J., Codling, E., 2018. Navigational efficiency in a biased and correlated random walk model of individual animal movement. Ecology 99 (1), 217-223.

Benhamou, S., 2004. How to reliably estimate the tortuosity of an animal's path: straightness, sinuosity, or fractal dimension? J. Theor. Biol. 229 (2), 209-220.

Benhamou, S., 2006. Detecting an orientation component in animal paths when the preferred direction is individual-dependent. Ecology 87 (2), 518-528.

Benhamou, S., 2018. Mean squared displacement and sinuosity of threedimensional random search movements. arXiv 1801.02435. Retrieved from: http://arxiv.org/abs/1801.02435.

Bonsall, M., Dooley, C., Kasparson, A., Brereton, T., Roy, D., Thomas, J., 2014. Allee effects and the spatial dynamics of a locally endangered butterfly, the high brown fritillary (argynnis adippe). Ecol. Appl. 24, 108-120. 
Bonsall, M., Froyd, C., Jeffers, E., 2020. Resilience: nitrogen limitation, mycorrhiza and long-term palaeoecological plant-nutrient dynamics. Biol. Lett. 16: 20190441. http://doi.org/10.1098/rsbl.2019.0441.

Bovet, P., Benhamou, S., 1988. Spatial analysis of animals' movements using a correlated random walk model. J. Theor. Biol. 131 (4), 419-433.

Brown, G., Matthews, I., 2016. A review of extensive variation in the design of pitfall traps and a proposal for a standard pitfall trap design for monitoring groundactive arthropod biodiversity. Ecol. Evol. 6 (12), 3953-3964.

Buckland, S., Rexstad, E., Marques, T., Oedekoven, C., 2015. Distance Sampling: Methods and Applications. Springer International.

Bullock, J., Kenward, R., Hails, R., 2002. Dispersal Ecology. Blackwell Science, Malden, Massachusetts, USA.

Byers, J., 2001. Correlated random walk equations of animal dispersal resolved by simulation. Ecology 82 (6), 1680-1690.

Byers, J., 2011. Analysis of vertical distributions and effective flight layers of insects: three-dimensional simulation of flying insects and catch at trap heights. Environ. Entomol. 40 (5), 1210-1222.

Byers, J., 2012. Estimating insect flight densities from attractive trap catches and flight height distributions. J. Chem. Ecol. 38 (5), 592-601.

Charnick, S., Lauffenburger, D., 1990. Mathematical analysis of cell-target encounter rates in three dimensions. effect of chemotaxis. Biophys. J. 57 (5), 1009-1023.

Clobert, J., Danchin, E., Dhondt, A., Nichols, J., 2001. Dispersal. Oxford University Press, Oxford.

Codling, E., Plank, M., 2011. Turn designation, sampling rate and the misidentification of power laws in movement path data using maximum likelihood estimates. Theor. Ecol. 4 (3), 397-406.

Codling, E., Plank, M., Benhamou, S., 2008. Random walk models in biology. J.R. Soc. Interface 5 (25). 813-834.

Crank, J., 1975. The Mathematics of Diffusion. Oxford University Press.

Daniel, C., Grunder, J., 2012. Integrated management of european cherry fruit fly rhagoletis cerasi (1.): situation in switzerland and europe. InSections 3(4), 956988.

de Margerie, E., Pichot, C., Benhamou, S., 2018. Volume-concentrated searching by an aerial insectivore, the common swift (apus apus). Anim. Behav. 136, 159172.

de Valpine, P., Hastings, A., 2002. Fitting population models incorporating process noise and observation error. Ecol. Monogr. 72 (1), 57-76.

Doucet, A., Nando de, F., Gordon, N., 2001. Sequential Monte Carlo Methods in Practice. Springer Verlag.

Edelhoff, H., Signer, J., Balkenhol, N., 2016. Path segmentation for beginners: an overview of current methods for detecting changes in animal movement patterns. Movement Ecol. 4 (21).

El-Sayed, A., Suckling, D., Byers, J., Jang, E., Wearing, C., 2009. Potential of lure and kill in long-term pest management and eradication of invasive species. J. Econ. Entomol. 102(3), 815-835.

El-Sayed, A., Suckling, D., Wearing, C., Byers, J., 2006. Potential of mass trapping for long term pest management and eradication of invasive species. J. Econ. Entomol. 99, 1550-1564.

Epsky, N., Morrill, W., Mankin, R., 2004. Traps for Capturing Insects. Springer, Dordrecht. In: Encyclopedia of Entomology.

Fisher, N., Lewis, T., Willcox, M., 1981. Tests of discordancy for samples from fisher's distribution on the sphere. J. Appl. Stat. 30 (3), 230-237.

Fortin, D., Morales, J., Boyce, M., 2005. Elk winter foraging at fine scale in yellowstone national park. Oecologia 145 (335-343).

Fukaya, K., Royle, J., 2013. Markov models for community dynamics allowing for observation error. Ecology 94 (12), 2670-2677.

Gerritsen, J., Strickler, J., 1977. Encounter probabilities and community structure in zoo-plankton: a mathematical model. J. Fish. Board Can. 34 (1), 73-82.

Greenslade, P., 1964. Pitfall trapping as a method for studying populations of Carabidae (Coleoptera). J. Animal Ecol. 33 (2), 301-310.

Grimmet, R., Stirzaker, D., 2001. Probability and Random Processes. Oxford University Press.

Gurarie, E., Ovaskainen, O., 2011. Characteristic spatial and temporal scales unify models of animal movement. Am. Natural. 178 (1), 113-123. 113-123.

Gurarie, E., Ovaskainen, O., 2013. Towards a general formalization of encounter rates in ecology. Theor. Ecol. 6, 189-202.

Hall, R., 1977. Amoeboid movements as a correlated walk. J. Math. Biol. 4, $327-335$.

Hilborn, R., Mangel, M., 1998. The Ecological Detective. Princeton University Press, Princeton, NJ.

ISPM 26., 2018. International Standard for Phytosanitary Measures. Establishment of pest free areas for fruit flies (Tephritidae). Food and Agriculture Organization of the United Nations (FAO). Produced by the Secretariat of the International Plant Protection Convention. Current adopted versions of ISPMs are available for download on www.ippc.int.

James, A., Plank, M., Edwards, A., 2011. Assessing Lévy walks as models of animal foraging. J. R. Soc. Interface 8 (62), 1233-1247. doi: 10.1098/rsif.2011.0200.

Johnson, D., London, J., Lea, M., Durban, J., 2008. Continuous-time correlated random walk model for animal telemetry data. Ecology 89, 1208-1215.

Kantas, N., Doucet, A., Singh, S., Maciejowski, J., Chopin, N., 2015. On particle methods for parameter estimation in state-space models. Stat. Sci. 30, 328-351.

Kareiva, P., Shigesada, N., 1983. Analyzing insect movement as a correlated random walk. Oecologia 56 (2-3), 234-238.

Katsoyannos, B., 1989. Response to shape, size and color. In: Robinson, A.S., Hooper, G. (Eds.), Fruit Flies Their Biology, Natural Enemies and Control. 3A. Elsevier, Amsterdam, The Netherlands, pp. 307-324.
Kot, M., 1992. Discrete-time travelling waves: ecological examples. J. Math. Biol. 30, 413-436.

Kot, M., Schaffer, W., 1986. Discrete-time growth-dispersal models. Math. Biosci. 80 (1), 109-136.

Kura, K., Khamis, D., Mouden, C., Bonsall, M., 2019. Optimal control for disease vector management in sit models: an integrodifference equation approach. J. Math. Biol. 78, 1821-1839.

Le Bras, Y., Joumaa, J., Guinet, C., 2017. Three-dimensional space use during the bottom phase of southern elephant seal dives. Movement Ecol. 5 (18).

Li, M., Bolker, B., 2017. Incorporating periodic variability in hidden markov models for animal movement. Movement Ecol. 5 (1).

Lin, C.C., Segel, L.A., 1974. Mathematics Applied to Deterministic Problems in the Natural Sciences. Macmillan, New York, NY.

Lutscher, F., 2019. Integrodifference Equations in Spatial Ecology. Springer.

Mardia, K., Jupp, P., 2000. Directional Statistics. John Wiley and Sons, Chichester.

Mardia, K., Kent, J., Bibby, J., 1979. Multivariate Analysis. Academic Press, San Diego.

Marsh, L., Jones, R., 1988. The form and consequences of random walk movement models. J. Theor. Biol. 133, 113-131.

Martinez-Garcia, R., Fleming, C., Seppelt, R., Fagan, W., Calabrese, J., 2020. How range residency and long-range perception change encounter rates. J. Theor. Biol. 498 (110267).

McClintock, B., King, R., Thomas, L., Matthiopoulos, J., McConnell, B., Morales, J., 2012. A general discrete-time modeling framework for animal movement using multistate random walks. Ecol. Monogr. 82, 335-349.

McClintock, B., Langrock, R., Gimenez, O., Cam, E., Borchers, D., Glennie, R., Patterson, T., 2020. Uncovering ecological state dynamics with hidden markov models. Ecol. Lett. 23, 1878-1903.

Miller, J., Adams, C., Weston, P., Schenker, J., 2015. Trapping of Small Organisms Moving Randomly. Principles and Applications to Pest Monitoring and Management. Springer. Springer briefs in ecology, United States.

Morales, J., Haydon, D., Frair, J., Holsinger, K., Fryxell, J., 2004. Extracting more out of relocation data: building movement models as mixtures of random walks. Ecology 85 (9), 2436-2445.

Muirhead-Thomson, R., 1991. Trap Responses of Flying Insects. Elsevier. The Influence of Trap Design on Capture Efficiency.

Nathan, R., Getz, W., Revilla, E., Holyoak, M., Kadmon, R., Saltz, D., 2008. A movement ecology paradigm for unifying organismal movement research. Proc. Natl. Acad. Sci. USA 105, 19052-19059.

Okubo, A., 1980. Diffusion and Ecological Problems: Mathematical Models. Springer, Berlin.

Owen-Smith, N., Fryxell, J., Merrill, E., 2010. Foraging theory upscaled: the behavioural ecology of herbivore movement. Philos. Trans. R. Soc. B 365, 2267-2278.

Patlak, C., 1953. Random walk with persistence and external bias. Bull. Math. Biophys. 15, 311-338.

Petrovskii, S., Bearup, D., Ahmed, D., Blackshaw, R., 2012. Estimating insect population density from trap counts. Ecol. Complexity 10, 69-82.

Petrovskii, S., Petrovskya, N., Bearup, D., 2014. Multiscale approach to pest insect monitoring: random walks, pattern formation, synchronization and networks. Phys. Life Rev. 11 (3), 467-525.

Pyke, G., 1984. Optimal foraging theory - a critical review. Annu. Rev. Ecol. Evol. Syst. $15,523-575$.

Pyke, G., 2015. Understanding movements of organisms: it's time to abandon the Lévy foraging hypothesis. Methods Ecol. Evol. 6 (1), 1-16.

Qin, Y., Paini, D., Wang, C., Fang, Y., Li, Z., 2015. Global establishment risk of economically important fruit fly species (tephritidae). PLoS One 10, (1) e0116424.

Radcliffe, E., Hutchison, W., Cancelado, R., 2008. Integrated Pest Management: Concepts, Tactics, Strategies and Case Studies. Cambridge University Press.

Redner, S., 2001. A Guide to First Passage Processes. Cambridge University Press.

Reimer, J., Bonsall, M., Maini, P., 2016. Approximating the critical domain size of integrodifference equations. Bull. Math. Biol. 78, 72-109.

Reimer, J., Bonsall, M., Maini, P., 2017. The critical domain size of stochastic population models. J. Math. Biol. 74, 755-782.

Reynolds, A., 2010. Bridging the gulf between correlated random walks and lévy walks: autocorrelation as a source of lévy walk movement patterns. J. R. Soc. Interface 7, 1753-1758.

Robacker, D., Rodriguez, M., 2004. A simple and effective cylindrical sticky trap for fruit flies (diptera: Tephritidae). Florida Entomol. 87 (4), 492-495.

Sadjadi, Z., Shaebani, M., Rieger, H., Santen, L., 2015. Persistent-random-walk approach to anomalous transport of self-propelled particles. Phys. Rev. E 91, (6) 062715.

Schultz, C., Crone, E., 2001. Edge-mediated dispersal behaviour in a prairie butterfly. Ecology 82, 1879-1892.

Siewers, J., Schirmel, J., Buchholz, B., 2014. The efficiency of pitfall traps as a method of sampling epigeal arthropods in litter rich forest habitats. Eur. J. Entomol. 111 (1), 69-74. https://doi.org/10.14411/eje.2014.008.

Sivinski, J., 1990. Colored spherical traps for capture of caribbean fruit fly, anastrepha suspensa. Florida Entomol. 73 (1), 123.

Skellam, J.G., 1951. Random dispersal in theoretical populations. Biometrika 38, $196-218$.

Sornette, D., 2004. Critical Phenomena in Natural Sciences. second ed., Berlin, Springer.

Southwood, T., 1978. Ecological Methods. Chapman and Hall, London.

Tchen, C., 1952. Random flight with multiple partial correlations. J. Chem. Phys. 20, 214. 
Thomas, C., Parkinson, L., Marshall, E., 1998. Isolating the components of activitydensity for the carabid beetle Pterostichus melanarius in farmland. Oecologia 116 (1-2), 103-112.

Tilles, P., Petrovskii, S., 2015. Statistical mechanics of animal movement: animals's decision-making can result in superdiffusive spread. Ecol. Compl. 22, 86-92.

Tilles, P., Petrovskii, S., 2019. On the consistency of the reaction-telegraph process within finite domains. J. Stat. Phys. 177, 569-587.

Tilles, P., Petrovskii, S., Natti, P., 2017. A random acceleration model of individual animal movement allowing for diffusive, superdiffusive and superballistic regimes. Sci. Rep. 7, 14364.

Turchin, P., 1998. Quantitative Analysis of Movement. Measuring and Modelling Population Redistribution in Animals and Plants. Sinauer Associates, Inc., Sunderland, Massachusetts.

Vargas, R., Piñero, J., Leblanc, L., 2015. An overview of pest species of bactrocera fruit flies (diptera: Tephritidae) and the integration of biopesticides with other biological approaches for their management with a focus on the pacific region. Insects 6 (2), 297-318.

Viswanathan, G., Afanasyev, V., Buldryrev, S., Havlin, S., da Luz, M., Stanley, H., 2011. The Physics of Foraging. Cambridge University Press.
Voesenek, C., Pieters, R., van Leeuwen, J., 2016. Automated reconstruction of threedimensional fish motion, forces, and torques. PLoS One 11, e0146682.

Wang, M., Kot, M., Neubert, M., 2002. Integrodifference equations, allee effects, and invasions. J. Math. Biol. 44 (2), 150-168.

Weiss, G.H., 1994. Aspects and Applications of the Random Walk. North Holland Press, Amsterdam, The Netherlands.

Williams, H., Taylor, L., Benhamou, S., Bijleveld, A., Clay, T., de Grissac, S., Demar, U., English, H., Franconi, N., GómezÿLaich, A., Griffiths, R., Kay, W., Morales, J., Potts, J., Rogerson, K., Rutz, C., Spelt, A., Trevail, A., Wilson, R., Börger, L., 2020. Optimising the use of biologgers for movement ecology research. J. Animal Ecol. 89, 186-206. https://doi.org/10.1111/1365-2656.13094.

Wilson, R., Griffiths, I., Legg, P., Friswell, M., Bidder, O., Halsey, L., Lambertucci, S., Shepard, E., 2013. Turn costs change the value of animal search paths. Ecol. Lett. 16 (9), 1145-1150.

Yee, W., 2019. Three-dimensional versus rectangular sticky yellow traps for western cherry fruit fly (diptera: Tephritidae). J. Econ. Entomol. 112 (4), $1780-1788$.

Zhou, Y., Kot, M., 2011. Discrete-time growth-dispersal models with shifting species ranges. Theor. Ecol. 4, 13-25. 